\title{
Effects of Pet Therapy in Elderly Patients with Neurocognitive Disorders: A Brief Review
}

\author{
Chiara Sbrizzi ${ }^{a}$ Walter Sapuppo ${ }^{a, b}$ \\ ${ }^{a}$ Department of Psychology, Sigmund Freud University, Milan, Italy; ${ }^{b}$ Division of Psychology, London South Bank \\ University, London, UK
}

\section{Keywords}

Pet therapy · Anima-assisted intervention · Neurocognitive disorder · Alzheimer · Geriatric

\begin{abstract}
Introduction: Neurocognitive disorders (NCDs) are disturbances highly related to age. This means that, with the increasing trend in life expectancy, there is also an increase in this diagnosis, although NCDs are not exclusively found in the population over 65 years old. Likely, they will increase in the coming years together with improvements in diagnosis. In addition to the use of medicines and rehabilitative techniques, pet therapy is also used. Pet therapy makes use of animals with therapeutic, rehabilitative, educational, and recreational purposes for people affected by physical, neuromotor, and psychiatric disorders. Pet therapy seems to be functional for increasing social and communication competencies, facilitating verbal and body language, increasing self-esteem, improving quality of life, and reducing anxiety/ stress. Methods: This study was based on scientific papers and publications obtained from the PubMed and Google Scholar databases. Moreover, other articles from further cross-references were included. Specific database research criteria were (a) articles published in 2018 or later, (b) samples containing only adults over 65 years old, (c) written in
\end{abstract}

English or Italian, and (d) on the topic of animal-assisted intervention. Results: Uncertain results were obtained. Although a positive effect was found, the included articles were of insufficient methodological rigor. Discussion/Conclusion: Although many studies reported positive results, these could not be generalized because of the numerous biases present (e.g., small sample size, lack of methodological rigor, lack of protocol, etc.). Future studies, therefore, should seek to address the limitations found in the analyzed studies.

(c) 2021 The Author(s).

Published by S. Karger AG, Basel

\section{Introduction}

As individuals age, they experience inevitable physiological and emotional/cognitive changes that can lead to changes in social interactions. In principle, all individuals over 65 years of age are defined as "elderly"; this age limit has been set quite arbitrarily [1]. Gerontologists generally divide the population over 65 years of age into 3 groups: the group between 65 and 74 years of age is defined as "young elders," the one between 75 and 84 years of age defined "elderly elders," and finally the last one defined by "ultra-elders" over 84 years of age [1].

karger@karger.com www.karger.com/dee

Karger $\stackrel{\text { ' }}{5}$
C) 2021 The Author(s).

Published by S. Karger AG, Basel

This is an Open Access article licensed under the Creative Commons Attribution-NonCommercial-4.0 International License (CC BY-NC) (http://www.karger.com/Services/OpenAccessLicense), applicable to the online version of the article only. Usage and distribution for commercial purposes requires written permission. 


\section{Epidemiology}

Mental health is related to physical and cognitive problems, which people can experience throughout their life. The elderly population experiences more problems than all other age-groups [1]. According to the National Academy on an Aging Society [2], 80\% of the elderly population suffers from at least one major medical condition. Neurocognitive disorders (NCDs) [3] are age-related disorders, and this means that as the years of age increase, there is also an increase in this kind of diagnosis; according to ISTAT (2014), about 600,000 people in Italy have a NCD due to Alzheimer's disease or dementia. Worldwide, life expectancy is increasing and with this also the elderly population; in 1990, $<200,000,000$ people were aged $\geq 65$ years, while current prognoses suggest that, in 2030, this population will exceed $1,300,000,000$, increasing overall by $180 \%$ [4]. In 2017, according to a World Health Organization (OMS) report, 5-8 out of 100 people are suffering from NCD, with some 50 million people affected globally [5]. It is estimated that the number of people with NCD worldwide will increase at a rate of 10 million per year and that the total population with a NCD will reach 82 million by 2030 and 152 million by 2050 [6]. The study of NCD shows an increase in cases even in youth, but it is undisputed that the majority of cases are observed in the population over 65 years of age. This phenomenon is related to the constant increase in the average life span of the population, which is also measured by the aging index, that is, the ratio between the number of elderly people (age $>65$ years) and the number of young people (age $<15$ years). In Italy, the aging index shows $>144$ elderly people for every 100 young people [7]. NCD is a pathology that involves the individual in its entirety (some of the areas involved are memory, language, thought, mobility, sleep-wake rhythm, attention, thought processing, etc.) [8] and becomes highly debilitating. From the pharmacological point of view, there are no products that can reverse this pathology, but current drug therapy can slow down the progress of the disorder by delaying degeneration and neuronal death [6].

\section{Pharmacotherapy}

The pharmacological approach does not aim to act on the causes of the disease, but rather on the evident symptoms of the patient. Generally, drugs act on the following functions: cognitive, behavioral, disability, and for the

Pet Therapy and Neurocognitive Disorders improvement of quality of life. To counteract the symptoms of NCD, anticholinesterase drugs and nootropic drugs are used [8]. In addition to using drugs to combat cognitive impairment in NCD, drugs are often used to mediate the symptoms of psychotic and behavioral disorders. Studies have confirmed that, during the disease, patients with NCD also show symptoms beyond the cognitive sphere, which are mainly responsible for burnout in caregivers [8]. Generally, psychopharmacological approach is adopted, that is, using a drug of known efficacy for the symptoms that are most similar to those presented by the patient. As mentioned above, for NCD, there is no cure with pharmacological treatments and they are not able to bring about symptomatic reduction of the disease to slow its progress. Also, drug treatments can have negative side effects [9]. For this reason, there is a need to implement nonpharmacological treatments in order to offer alternatives [10].

\section{Pet Therapy}

In the field of nonpharmacological therapies, pet therapy can also be a good way forward. This nonpharmacological therapy is defined as a useful, versatile, and potentially effective tool, also in terms of cost, to mitigate the symptoms of the disorder and to improve the quality of life of subjects with NCD and their caregivers [11]. This is due to the fact that, with the worsening of NCD, the ability to communicate verbally and comprehension is reduced, but through pet therapy, communication can be stimulated despite these difficulties. This is due to the reception and interpretation of stimuli and signals such as visual, tactile, auditory, and olfactory. In the elderly, the presence of animals can often evoke positive memories, for example, links with animals they had previously [12]. A study by Odendaal and Meintjes [13] observed that contact with animals leads to increased concentrations of endorphins, oxytocin, prolactin, phenylethylamine, and dopamine and reduced plasma cortisol levels. Oxytocin has widespread neurological, biological, emotional, and social effects, including attachment and social processes, decreases anxiety, stress, and aggressive behavior [14]. It also stimulates the parasympathetic nervous system, reduces the secretion of cortisol, aldosterone and adrenaline, increases pain tolerance, lowers blood pressure, decreases inflammation, improves wound healing, and facilitates learning [15].

The primary objective of this work was to understand the effects of pet therapy in elderly patients with NCD. 


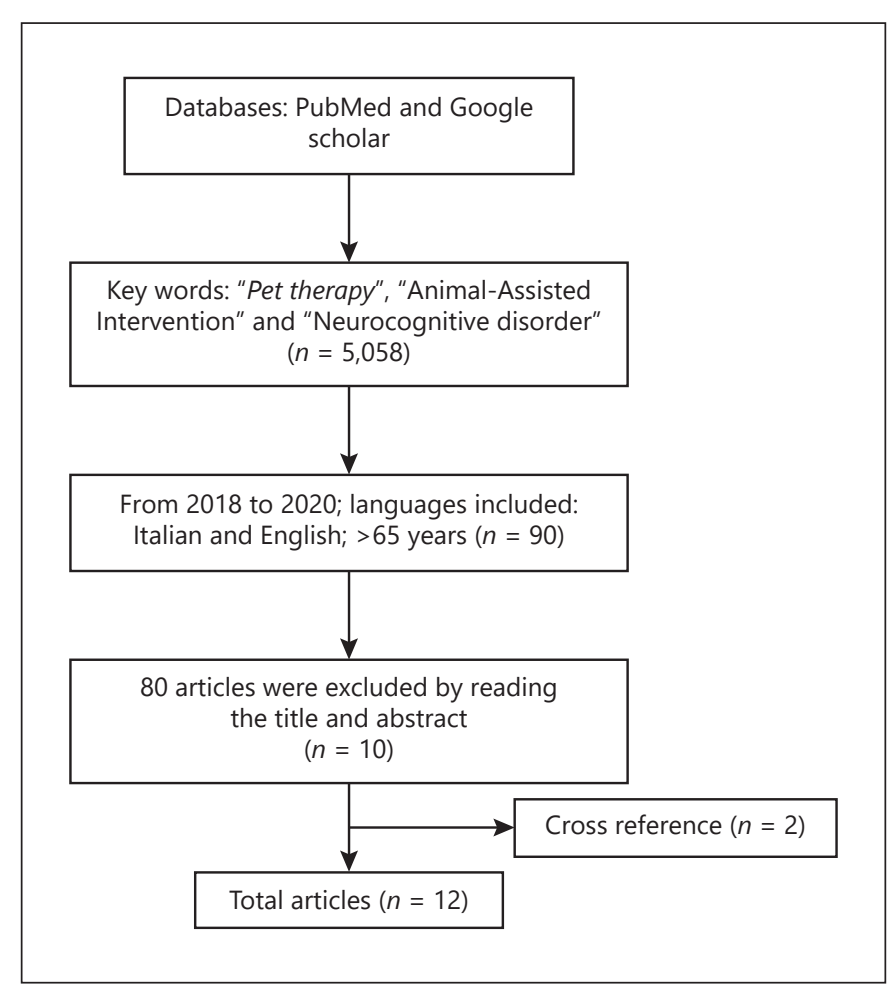

Fig. 1. PRISMA: Search and selection guidelines flow chart.

Another aim was to provide a further empirical basis for this topic and to identify the obstacles encountered so far in research, in the hope of providing further elements to help overcome them in future studies.

\section{Methods}

The material for the systematic review of the literature on pet therapy and NCD was found in the databases Google Scholar and PubMed; the words used were "Animal assisted interventions" AND "Pet therapy" AND "Neurocognitive disorder" AND "Over 65s." The PRISMA method was used to find the articles. The articles must meet the following inclusion criteria: a limit was set for the publication year, conventionally defined as 2018 (the date of the first meta-analysis dealing with animal assisted intervention [AAI] in general), which allowed us to analyze the most recent findings on the subject (from 2018 to 2020, inclusive). The articles must deal mainly with assisted interventions with animals as the topic, written in English or Italian, and concern a population of elderly people only (i.e., 65 years old or older). Although NCD can also affect younger individuals, NCD primarily involves this type of population. Articles using any type of animal were included. The list of studies identified by the search engines was screened by reading (by the researcher CS) the titles and abstracts of the studies. Once this step was taken, the results of searches in the 2 databases were integrated. In the first phase of research, 5,058 articles were selected, and at the end of the selection process, a total of 12 articles were obtained. The articles were skimmed using the abovementioned criteria together with the reading of the titles and abstracts' an additional 2 articles that met the inclusion criteria were found through cross-referencing. The flowchart (Fig. 1) shows the whole skimming process, while Table 1 describes the articles included in this review. In addition, the distribution of the countries where the studies were conducted is shown in Figure 2.

\section{Results}

In the selected studies included within this review, the following areas were analyzed: quality of life $(n=7)$, mood (e.g., depression and emotional well-being; $n=6$ ), social function $(n=6)$, Behavioral and Psychological Symptoms of Dementia score $(n=3)$, symptoms closely related to $\operatorname{NCD}(n=3)$, agitation and irritability $(n=5)$, cognitive state $(n=8)$, physical functions (e.g., motor activities, daily activities, diet, and sleep; $n=5$ ), and adverse effects $(n=3)$. One study took into account the severity of the disease, mortality, and stress to the caregiver and the animal. These studies indicated a positive effect of the use of pet therapy; for example, the study conducted by Peluso et al. [19] states how human-animal interaction has positive effects by promoting self-esteem, improving cognitive performance such as memory or concentration, motor skills, and quality of life, and also decreases the effects of symptoms due to NCD. A study by Wesenberg et al. [12] also showed that the use of AAI leads to an increase in well-being in nursing home residents with NCD, increasing social interaction and emotional well-being. However, in some studies, there were no significant changes with respect to the areas investigated, but this aspect can be considered a remarkable achievement gave the progressive nature of the disorder [18]. However, despite the positive expectations, there was a common thread to all of the studies considered, which is the fact that the research was performed with a high degree of bias and deficits in research design and, consequently, in the execution. Because the results of the various existing studies are heterogeneous with considerable variation in study design, interventions, participants, baseline reports, and outcome measures, this makes comparison between studies difficult [12]. This is one of the main components that make it impossible to provide valid and certain conclusions about the effects of treatment through AAI, as it leads to controversial and ambiguous conclusions [19]. 


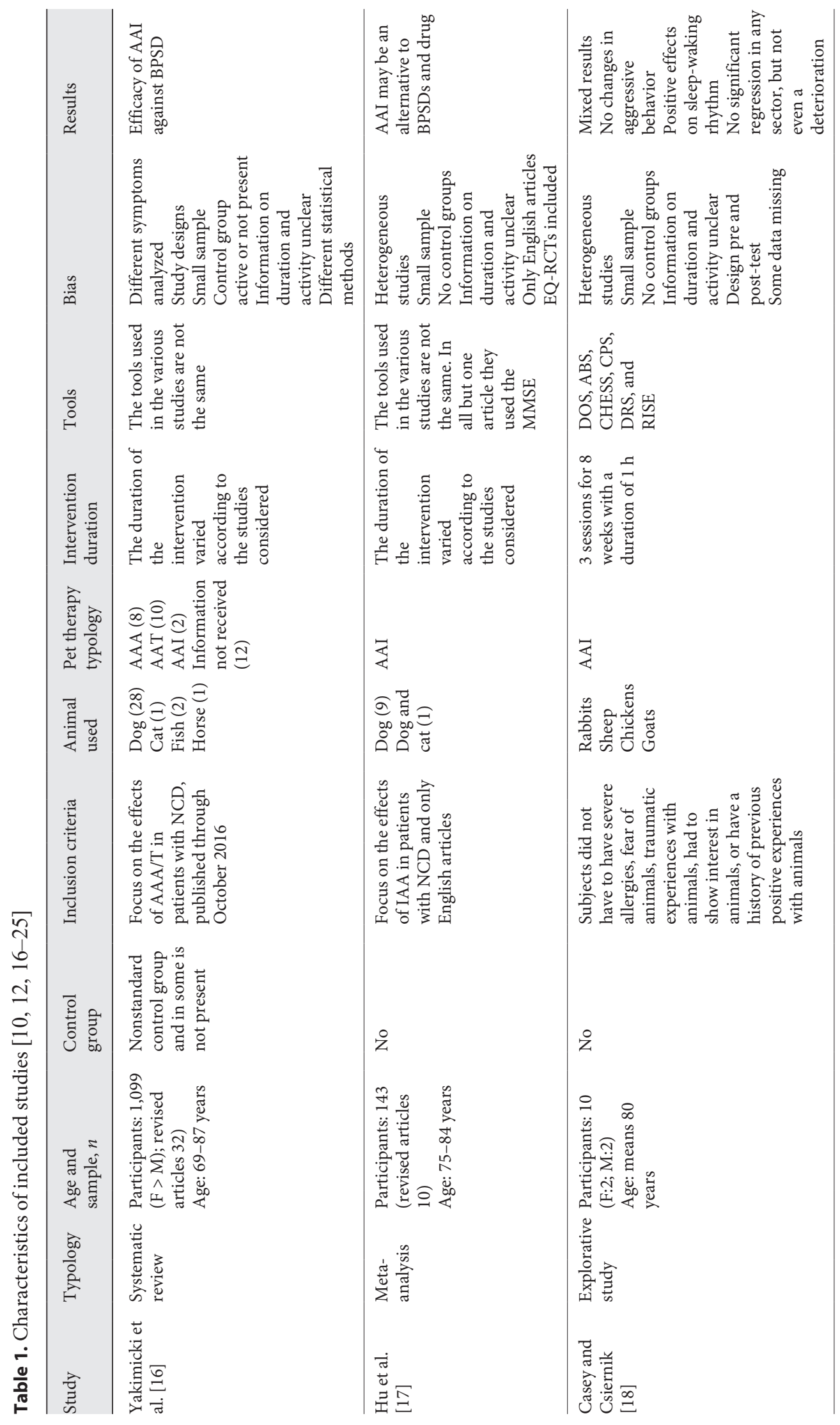




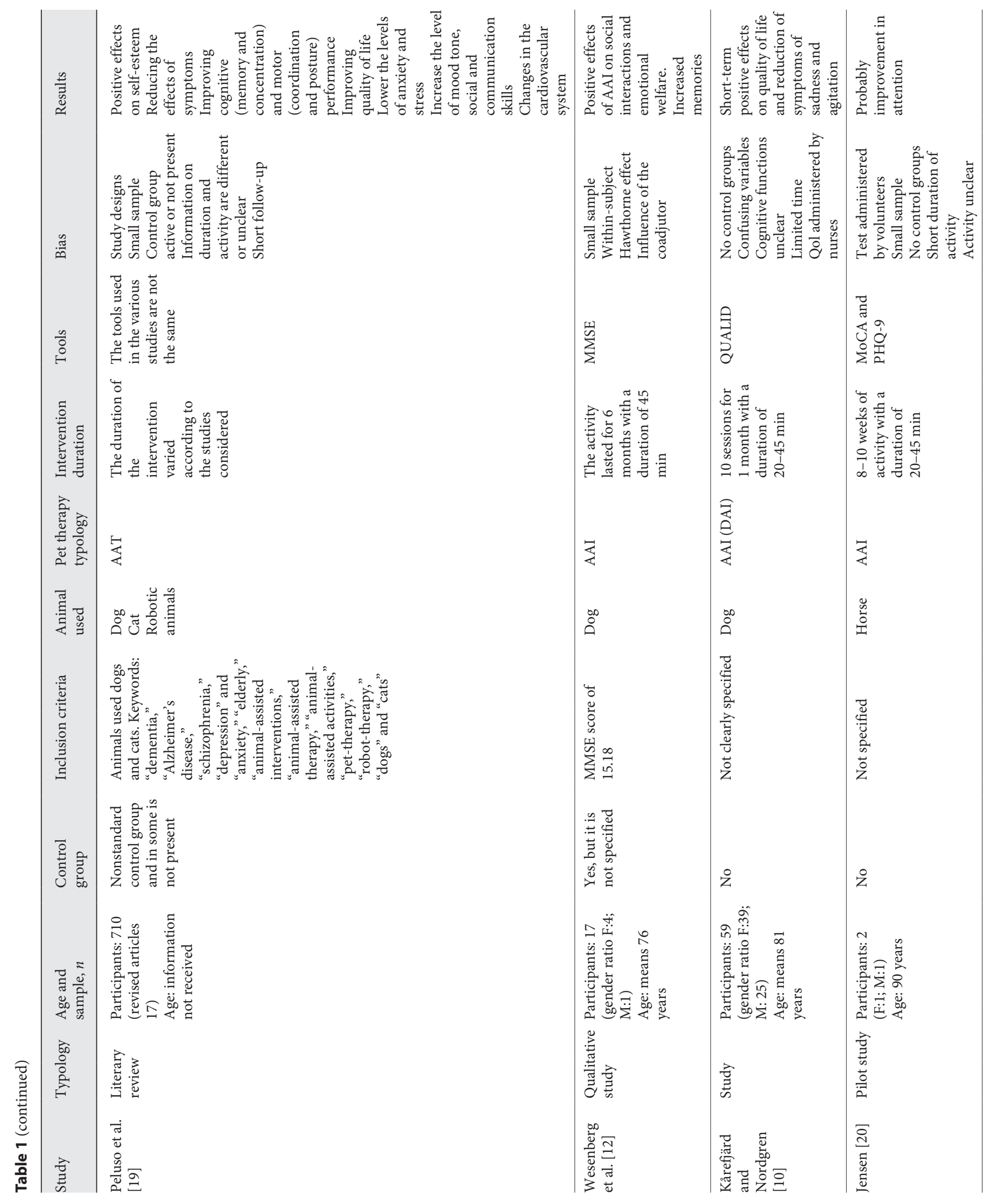




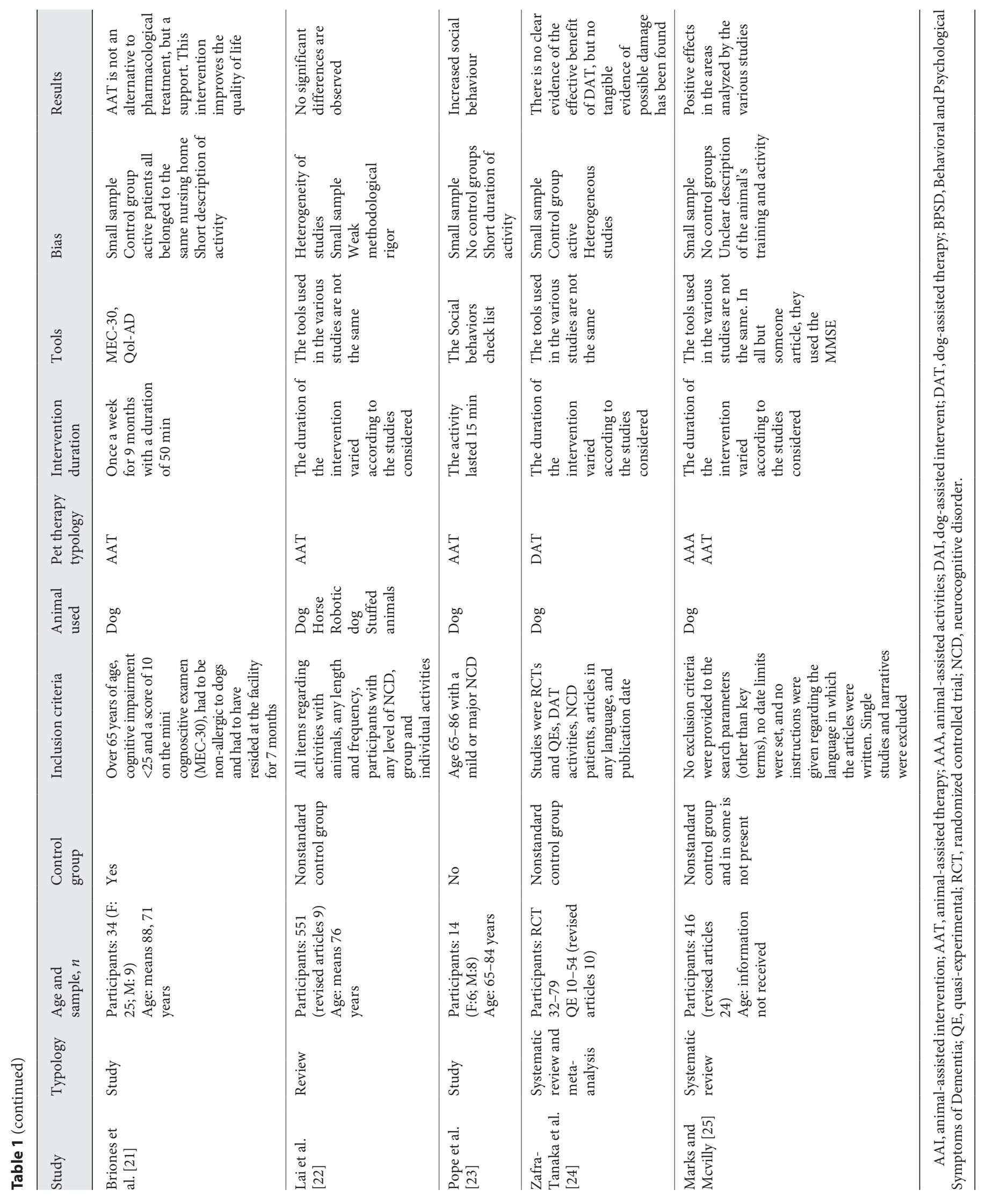


Fig. 2. Distribution of the countries where the studies were conducted.

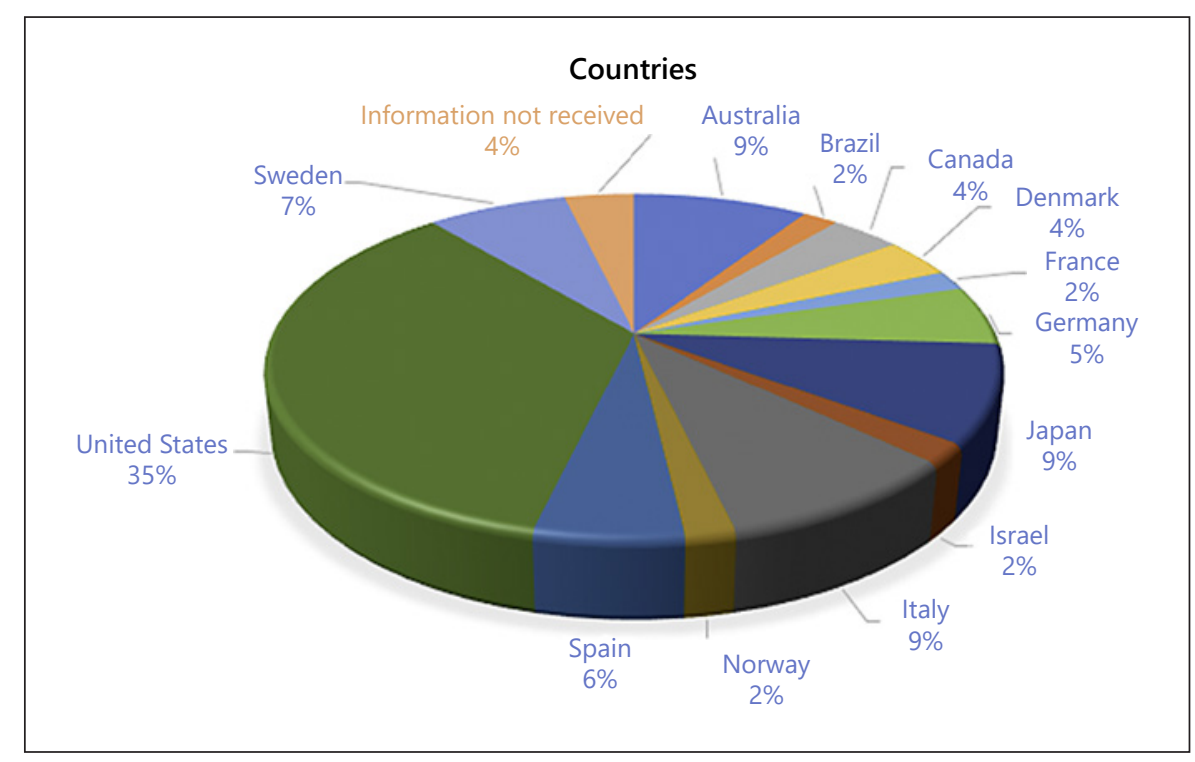

\section{Discussion/Conclusion}

The topic covered in this article shows the willingness and need to develop new methods of intervention for alternative NCD and/or support for other types of treatment. Pet therapy applied to NCD is an extremely recent intervention of psychosocial nature and still in the experimental phase; consequently, it is not very widespread in the academic and clinical world. Since NCD affect a large part of the population, this implies a waste of resources, time, and money for its cure. The total social cost of DNC is estimated about USD 820 billion, or $1.1 \%$ of the global gross domestic product [26] and, taking into account that in recent years an increase in this disease has been observed, it can be assumed that there has been a corresponding increase in health-care costs.

For this reason, it is also important to consider this aspect and understand whether AAI is a resource or an aggravation of costs. In recent years, there has been growing interest in research in this field [12], but, despite this, the state of the art of AAI research applied to NCD is still underdeveloped. The reasons for these difficulties are many and among the main one we can include: the obtained results, the methodological rigor, the timing of implementation, and the possibility of carrying out further research. NCD will certainly be one of the most significant health problems [21] in coming years, both in terms of health costs and the well-being of the person. As already pointed out, advancing age increases the risk of finding an NCD. The projection of statistical data indicates an increase in the diagnosis of this pathology in people over 65 years of age. Another relevant aspect is that NCD is not curable, but only the symptoms of this disease can be treated [7], which we have seen involve the functions of the individual as a whole.

Generally, the most commonly used treatments are pharmacological, which most often leads to side effects [10]. No treatment that is clearly and consistently effective in preventing or stopping the progression of this disorder has yet been identified [27]. For these reasons, alternative psychosocial interventions that avoid the use of drugs are being sought, and the World Health Organization also proposes the creation of plans and programs to improve the social well-being and quality of life of all those affected by NCD and their caregivers [21]. For this reason, one of the possibilities explored is the use of therapy animals, which is generating considerable interest in education and health services [25]. Pet therapy, as a type of intervention, is not intended to cure the disease, given the progressive form of the disorder. It is rather a treatment that can be relied on to induce relief to people who are affected and those who care for them, as stated in a study by Hu et al. [17]: "our results are not surprising because dementia is a progressive brain disease and it would be unrealistic to expect that the AAI reverses the cognitive state in a normal state." Observing the results of these studies clearly shows the mixed nature of the conclusions. From many kinds of studies, the positive character of the use of pet therapy has emerged. For example, the study 
conducted by Peluso et al. [19] states that the interaction between people and animals has positive effects in terms of promoting self-esteem, improving cognitive performance such as memory or concentration, motor skills, and quality of life while decreasing the effects of symptoms due to NCD. Also, the study by Wesenberg et al. [12] showed that the use of AAI leads to increased well-being in residents of nursing homes with NCD, increasing social interaction, and emotional well-being. If in some studies there were no significant changes compared to the investigated areas, this can also be considered a remarkable result given the progressive characteristic of the disorder [18]. However, despite the positive expectations, there was a common thread to all of the studies considered, which is the fact that the research was performed with a high degree of bias and deficits in research design and, consequently, in the execution. Because the results of the various existing studies are heterogeneous with considerable variation in study design, interventions, participants, baseline reports and outcome measures, this makes comparison between studies difficult [12]. This is one of the main components that make it impossible to provide valid and certain conclusions about the effects of treatment through AAI, as it leads to controversial and ambiguous conclusions [19].

Although many studies report positive results, these cannot be generalized because of the numerous biases present (e.g., small sample, lack of methodological rigor, lack of protocol, etc.). Nevertheless, these results and the urgent need to identify strategies to support drug treatment and to mitigate the side effects of drugs suggest implementing research around this topic. Future studies, therefore, should seek to address the limitations found in the analyzed studies.

\section{Limits}

Some of the limitations found are (i) many of the studies do not provide details on the implementation of the interventions, (ii) they either do not have a control group or the control group is also an active group (subjected to other types of interventions or they had unanticipated interactions with the animal), (iii) many confounding variables such as medication intake are present, (iv) timelines are short, (v) the sample size is small, (vi) dropouts occur during the trial (due to death, relocation or reluctance to continue), (vii) the sample is drawn from the same facility, (viii) there is no indication of the breed of the animal and its training, (ix) there is no consideration of whether the presence of the coadjutor would affect the outcome of the encounters, and (x) the methods of including participants are often unclear. Based on all these factors, it is very difficult to provide consistent and effective conclusions, but by introducing protocols and increasing methodological rigor, these limitations could be overcome.

\section{Conflict of Interest Statement}

The authors declare that they have no conflict of interest.

\section{Funding Sources}

This research has not been supported by funding.

\section{Author Contributions}

Both authors give substantial contributions to the conception or design of the work and to the interpretation of data. C.S. drafting the work and W.S. revising it critically and both give the approval of the version to be published.

\section{References}

1 Kring A, Conti D, Johnson S, Davison G, Neale J. Psicologia clinica. Bologna: Zanichelli; 2017.

2 National Academy on an Aging Society. Challenges for the 21st century: chronic and disabling conditions. 1999. Retrieved from: http: //www.agingsociety.org/agingsociety/publications/chronic/index.html.

3 American Psychiatric Association. Diagnostic and statistical manual of mental disorders; 2013.

4 Pierri G, Viola M. Incidenza della demenza in Italia. Rivista di psichiatria. 2003;38:6.

5 World Health Organization. Dementia. Factsheet. Geneva: World Health Organization [up- dated 2017 Dec]. Available from: www.who.int/ news-room/fact-sheets/detail/dementia.

6 World Health Organization. Overview of the global situation. Global action plan on the public health response to dementia 20172025. Geneva: World Health Organization; 2017. p. 2

7 Rovetto F. Psicologia clinica, psichiatria, psicofarmacologia. Milano: F. Angeli; 2015.

8 Cassano GB. Psicopatologia e clinica psichiatrica. Milano: Edra S.p.a.; 2015.

9 Hales RE, Yudofsky SC, Roberts LW. Manuale di psichiatria: American psychiatric publishing. In Weiner MF, editor. Disturbi neurocognitivi. Milano: Edra; 2015. p. 709-39.
10 Kårefjärd A, Nordgren L. Effects of dog-assisted intervention on quality of life in nursing home residents with dementia. Scand J Occup Ther. 2019 Oct;26(6):433-40.

11 Olazarán J, Reisberg B, Clare L, Cruz I, PenaCasanova J, del Ser T, et al. Eficacia de las terapias no farmacol.gicas en la enfermedad de alzheimer: revisión sistemática. Dement Geriatr Cogn Disord. 2010;30(2):161-78.

12 Wesenberg S, Mueller C, Nestmann F, Holthoff-Detto V. Effects of an animal-assisted intervention on social behaviour, emotions, and behavioural and psychological symptoms in nursing home residents with dementia. Psychogeriatrics. 2018;19(3):219-27. 
13 Odendaal JS, Meintjes RA. Neurophysiological correlates of affiliative behaviour between humans and dogs. Vet J. 2003;165(3):296301.

14 Netherton E, Schatte D. Potential for oxytocin use in children and adolescents with mental illness. Hum Psychopharmacol. 2011;26(45):271-81.

15 Chandler CK. Animal assisted therapy in counseling. New York: Routledge; 2005.

16 Yakimicki ML, Edwards NE, Richards E, Beck AM. Animal-assisted intervention and dementia: a systematic review. Clin Nurs Res. 2018;28(1):9-29.

17 Hu M, Zhang P, Leng M, Li C, Chen L. Animal-assisted intervention for individuals with cognitive impairment: a meta-analysis of randomized controlled trials and quasi-randomized controlled trials. Psychiatry Res. 2018; 260:418-27.

18 Casey J, Csiernik R. Farm animal assisted intervention with individuals with dementia: an Exploratory Study. ijrh. 2018;6(1):15-21.
19 Peluso S, De Rosa A, De Lucia N, Antenora A, Illario M, Esposito M, et al. Animal-assisted therapy in elderly patients: evidence and controversies in dementia and psychiatric disorders and future perspectives in other neurological diseases. J Geriatr Psychiatry Neurol. 2018;31(3):149-57.

20 Jensen C. Silver saddles: an equestrian intervention for older adults with dementia. Age Action. 2018;33(4):1-6. VCU Scholars Compass. Available from. https://scholarscompass.vcu.edu/vcoa_case/84/. Accessed 7 April 2020.

21 Briones MÁ, Pardo-García I, Escribano-Sotos F. Effectiveness of a dog-assisted therapy program to enhance quality of life in institutionalized dementia patients. Clin Nurs Res. 2021; 30(1):89-97.
22 Lai NM, Chang SMW, Ng SS, Tan SL, Chaiyakunapruk N, Stanaway F. Animal-assisted therapy for dementia. Cochrane Database Syst Rev. 2019;2019(11):CD013243.

23 Pope WS, Yordy M, Wang C. The effect of animal assisted therapy on participants with dementia in a community respite program. J Nurs Educ Pract. 2019;9(5).

24 Zafra-Tanaka JH, Pacheco-Barrios K, Tellez WA, Taype-Rondan A. Effects of dog-assisted therapy in adults with dementia: a systematic review and meta-analysis. BMC Psychiatry. 2019;19(1):41.

25 Marks G, Mcvilly K. Trained assistance dogs for people with dementia: a systematic review. Psychogeriatrics; 2020.

26 Prince M, Wimo A, Guerchet M, Ali GC, Wu YT, Prina M. World alzheimer report 2015: the global impact of dementia. Alzheimers Dis Intern. 2015. Retrieved from: https:// www.alz.co.uk/research/world-report-2015.

27 Schwarz S, Froelich L, Burns A. Pharmacological treatment of dementia. Curr Opin Psychiatry. 2012;25(6):542-50. 